\title{
Trends in prevalence, incidence and mortality of diagnosed and silent coronary heart disease in Quebec
}

\author{
C. Blais, PhD (1, 2); L. Rochette, MSc (1)
}

This article has been peer reviewed.

Tweet this article

\begin{abstract}
Introduction: Of all cardiovascular causes of mortality, coronary heart disease (CHD) remains the leading cause of death. Our objectives were to establish trends in the prevalence and incidence of CHD in the province of Quebec, and to determine the proportion of CHD mortality that had no previous CHD diagnosis.
\end{abstract}

Methods: Trends in prevalence, incidence and mortality were examined with a population-based study using the Quebec Integrated Chronic Disease Surveillance System, which links several health administrative databases. Data are presented using two case definitions for Quebecers aged 20 years and over: 1) a validated definition, and 2) CHD causes of death codes added to estimate the proportion of deaths that occurred without any previous CHD diagnosis as a proxy for sudden cardiac death (SCD).

Results: In 2012/2013, the crude prevalence of CHD was $9.4 \%$ with the first definition (593 000 people). Between 2000/2001 and 2012/2013, the age-standardized prevalence increased by $14 \%$, although it has been decreasing slightly since 2009/2010. Agestandardized incidence and mortality rates decreased by $46 \%$ and $26 \%$ respectively, and represented a crude rate of 6.9 per 1000 and $5.2 \%$ in 2012/2013. The proportion identified only by CHD mortality, our SCD proxy, was only significant for the incident cases (0.38 per 1000 in 2009/2010) and declined over the study period.

Conclusion: The prevalence of $\mathrm{CHD}$ has tended to decrease in recent years, and incidence and mortality have been declining in Quebec. Most CHD mortality occurs in previously diagnosed patients and only a small proportion of incident cases were not previously identified.

Keywords: coronary heart disease, trends, epidemiology, incidence, sudden cardiac death

\section{Introduction}

Although coronary heart disease (CHD) remains the worldwide leading cause of death, cardiovascular mortality in highincome countries is declining. ${ }^{1,2}$ This downward trend has been extensively studied. ${ }^{3-5}$ In Canada, the proportion associated with the decrease in cardiovascular risk factors (primary prevention) was $48 \%$, and the one associated with advances in medical and surgical treatments (secondary prevention) was $43 \%{ }^{6}$ However, recent epidemiological studies have demonstrated that primary prevention of CHD would be more difficult to implement because of the increasing prevalence of risk factors such as hypertension, ${ }^{7,8}$ diabetes ${ }^{9}$ and obesity, ${ }^{10}$ which could contribute to an increase in cardiovascular burden.
Key findings

- As Quebec is one of the few provinces in Canada that can link vital statistics with other health administrative data, the data can tell us whether people who died from coronary heart disease (CHD) had been previously diagnosed.

- Our results show that most of the people who died from CHD had been previously diagnosed with the disease. Only a small proportion of undiagnosed incident cases died from CHD.

- The burden of both diagnosed and silent CHD in Quebec is decreasing.

- Men had a higher prevalence and incidence, while mortality rates were the same for both sexes.

Many studies have addressed the public health burden of CHD death as well as of out-of-hospital CHD death, a surrogate for sudden cardiac death (SCD). ${ }^{11}$ These studies have documented evidence of a significant decrease in SCD rates. ${ }^{12,13}$ However, there is little information about the burden and trends of CHD mortality outside of hospitals and without any previous CHD diagnosis. A recent study revealed modest improvements over time in risk factor profiles of patients without known cardiovascular disease who presented with a first myocardial infarction. ${ }^{14}$ Because ventricular arrhythmias are life-threatening complications of acute myocardial infarction that are relatively common among people with no prior history of $\mathrm{CHD},{ }^{15}$ documenting the incidence of CHD mortality without prior CHD diagnosis would be useful. Chugh et al. $^{16}$ (p. 219) went further and mentioned that there exists "a critical need to learn more about patients who suffer SCD 
FIGURE 1

Case definitions of CHD plus the addition of CHD as cause of death to identify silent cases and their relationship with primary and secondary prevention

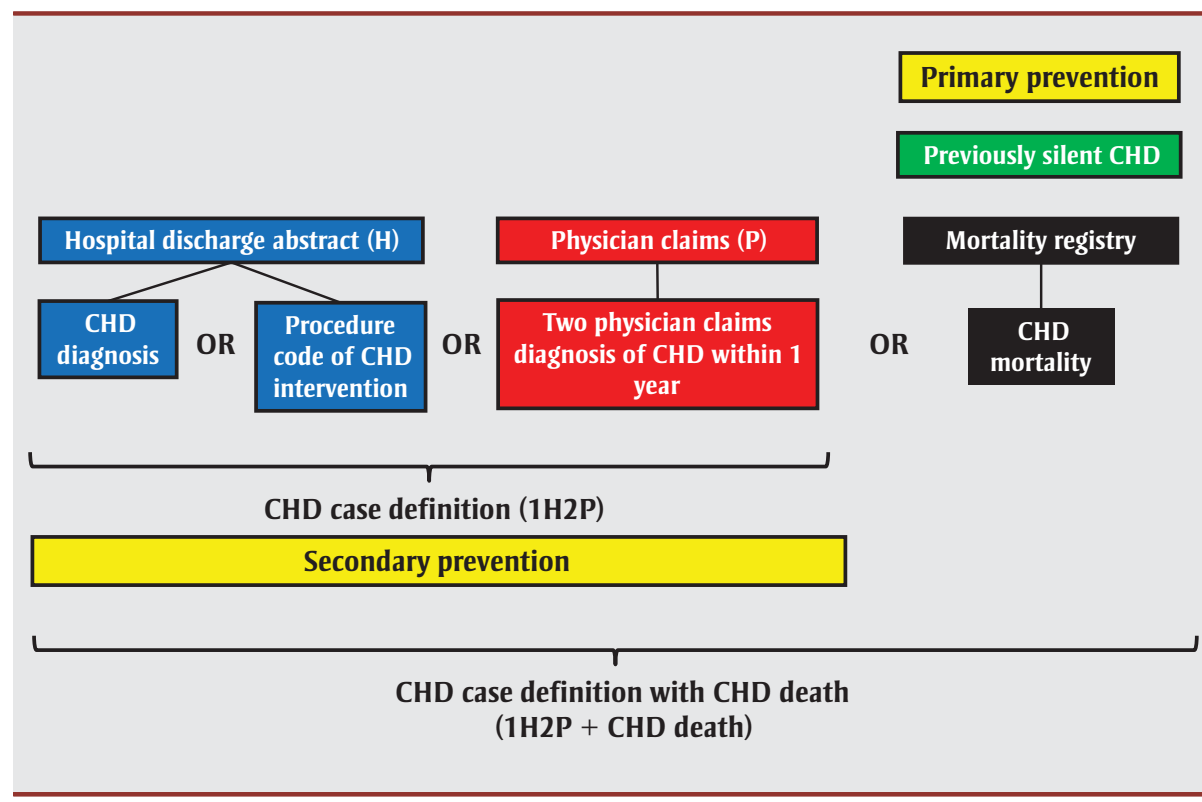

Abbreviations: $1 \mathrm{H} 2 \mathrm{P}$, one hospital or $\geq 2$ physician claims; CHD, coronary heart disease.

\section{FIGURE 2A}

Age-standardized ${ }^{\mathrm{a}}$ prevalence of diagnosed coronary heart disease, by sex and for all adults $\geq 20$ years, Quebec, 2000/2001 to 2012/2013, plus cases identified with coronary heart disease death

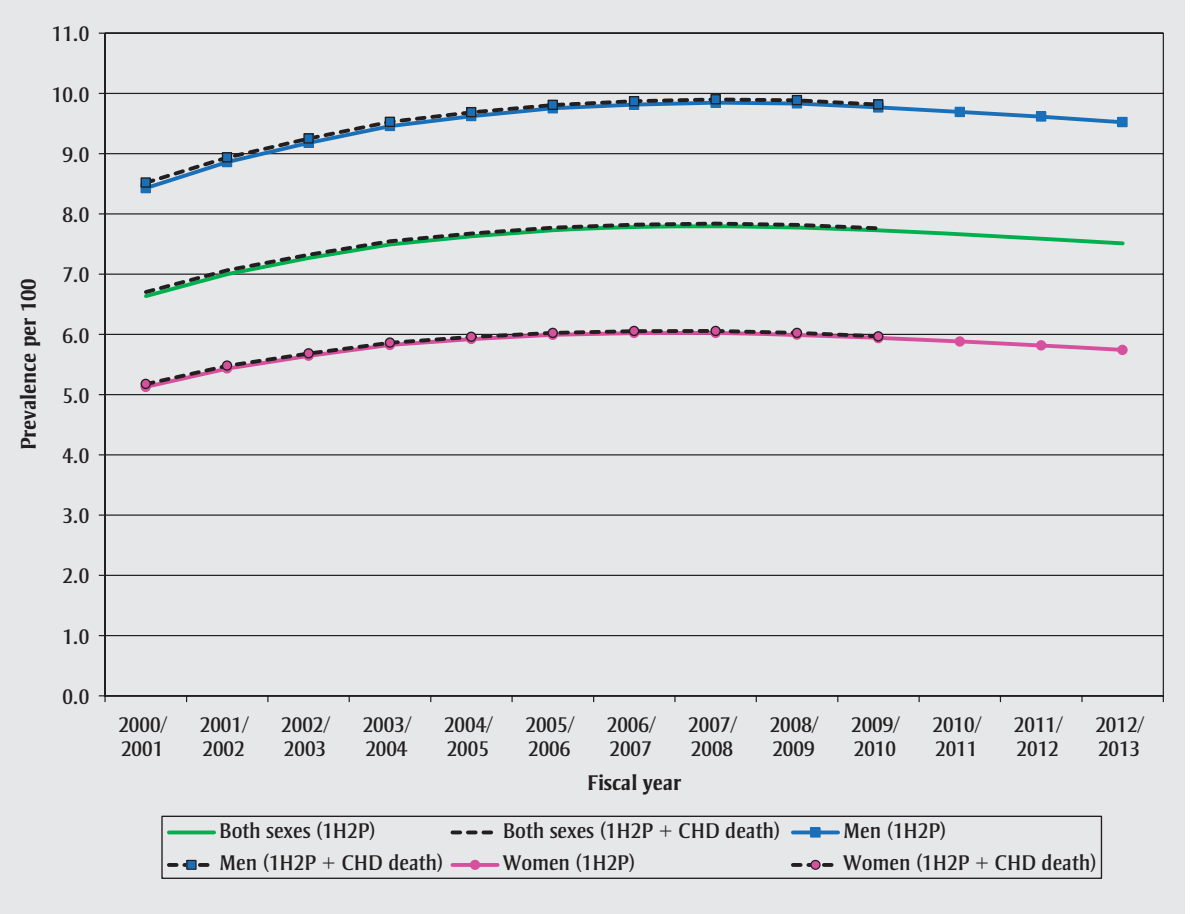

Source: Quebec Integrated Chronic Disease Surveillance System (QICDSS) of the Institut national de santé publique du Québec. Abbreviations: $1 \mathrm{H} 2 \mathrm{P}$, one hospital or $\geq 2$ physician claims; CHD, coronary heart disease.

${ }^{\text {a }}$ Age standardization using 2001 Quebec Census data as the standard population. in the community, particularly when they do not have previously identified heart disease."

Knowing that both primary and secondary prevention have greatly improved the trends in cardiovascular mortality, our first objective was to determine the trends in CHD prevalence, incidence and mortality in the province of Quebec, by sex and age, using a validated case definition. Our second objective was to determine the proportion of $\mathrm{CHD}$ mortality that occurred without any previous CHD diagnosis or treatment, a proxy of SCD, and to establish the trends in this proportion for prevalence, incidence and mortality in the last decade in Quebec, by sex and age.

\section{Methods}

\section{Data sources}

Our data source was the Quebec Integrated Chronic Disease Surveillance System (QICDSS), developed by the Institut national de santé publique du Québec. ${ }^{17}$ Briefly, the QICDSS was created by linking five health administrative databases: the health insurance registry, hospital discharges, physician claims, vital statistics and drug databases ( $\geq 65$ years; not used in this study). The QICDSS covers the entire Quebec population (8 million in 2012 in the health insurance registry) since $1^{\text {st }}$ January, 1996, and is updated annually, except for the mortality database, which is delayed; each fiscal year starts in April. The last year of mortality data we used was 2009/2010, while we used $2012 / 2013$ for the other databases.

\section{Coronary heart disease case definitions}

We used two case definitions for adults aged 20 years and over who are eligible for health insurance in Quebec: one for those diagnosed with $\mathrm{CHD}$ and the other that added people who received a CHD diagnosis only at death.

People were considered to be diagnosed with a CHD if they had received (1) a hospital discharge abstract with a principal or secondary CHD diagnosis code using International Classification of Diseases (ICD); (2) a hospital procedure code in any field of coronary intervention (coronary artery bypass graft or percutaneous coronary intervention); or (3) at least two physician claims with a CHD 


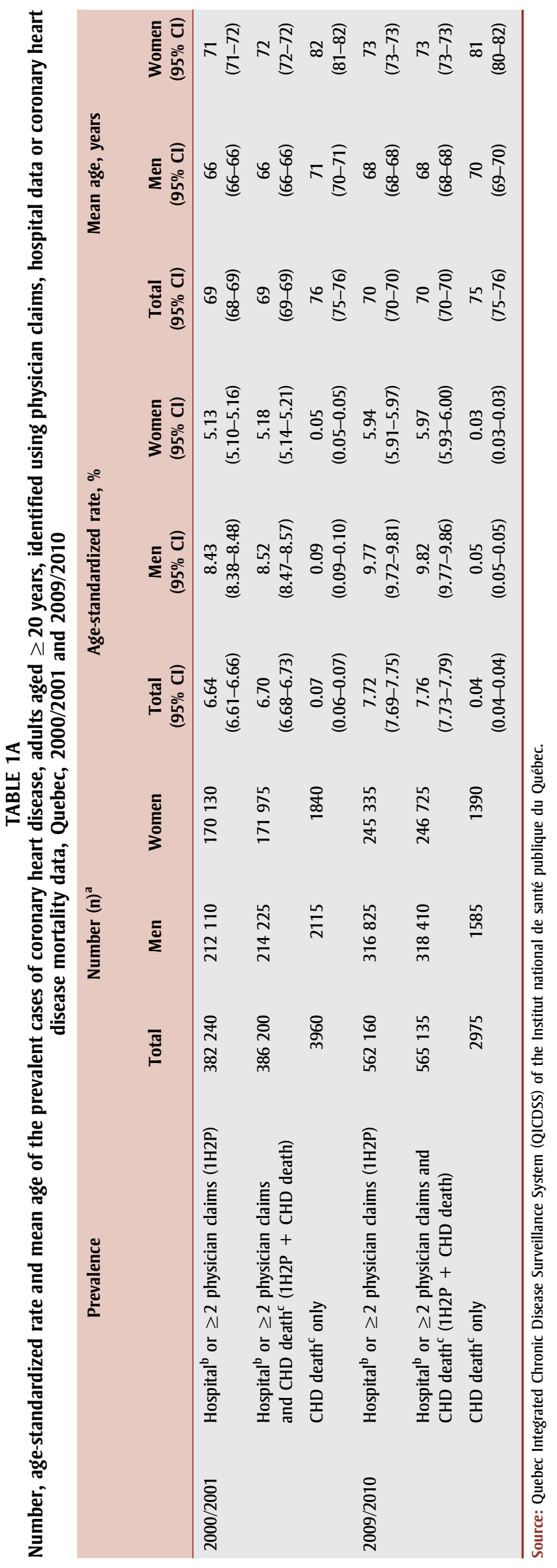

diagnosis code within a one-year period. The use of ICD and procedures codes has been described elsewhere. ${ }^{18}$ This first case definition, with slight modifications in the procedure codes, has been validated by Tu et al., ${ }^{19}$ and is used in the surveillance of CHD by the Canadian Chronic Disease Surveillance System, a collaborative network of provincial and territorial surveillance systems supported by the Public Health Agency of Canada. ${ }^{18}$ We call this case definition "1H2P."

For the second case definition, the mortality records data were added to $1 \mathrm{H} 2 \mathrm{P}$ to include the number of Quebecers who died of CHD but who had not been previously identified in the hospital or physician claims data (see Figure 1). This "1H2P + CHD death" case definition was applicable when the initial or any secondary causes of death corresponded to the $\mathrm{CHD}$ diagnosis codes referenced above. ${ }^{18}$ In other words, as the QICDSS links with the mortality records, $\mathrm{CHD}$ as cause of death is another possible way to identify cases of people without a diagnosis who died from CHD.

\section{Statistical analysis}

We calculated prevalence, incidence and mortality of CHD as previously described $^{8,18,20}$ with the number of eligible people in the health insurance registry as the denominator. Prevalent cases remained for the remainder of the follow-up period, as long as they were alive at the beginning of the year studied and had a valid health insurance card. We calculated prevalence by dividing the total number of prevalent cases by the insured population and multiplying by 100 . To calculate incidence, we divided the total number of newly diagnosed (incident) cases by the insured population at risk (total number of insured population minus the prevalent cases at the beginning of the fiscal year) and multiplied by 1000 . Because a minimum of four years was necessary to differentiate incident from prevalent cases, measures were reported from 2000/2001 even though observation began in January 1996.

We calculated mortality rates as the number of deaths from all causes among CHD patients divided by the number of $\mathrm{CHD}$ prevalent cases and multiplied by 100 . To analyze time trends, we used age-standardized rates and 
FIGURE 2B

Age-standardized prevalence of diagnosed coronary heart disease, by age group, in adults $\geq 20$ years, Quebec, 2000/2001 to 2012/2013, plus cases identified with coronary heart disease death

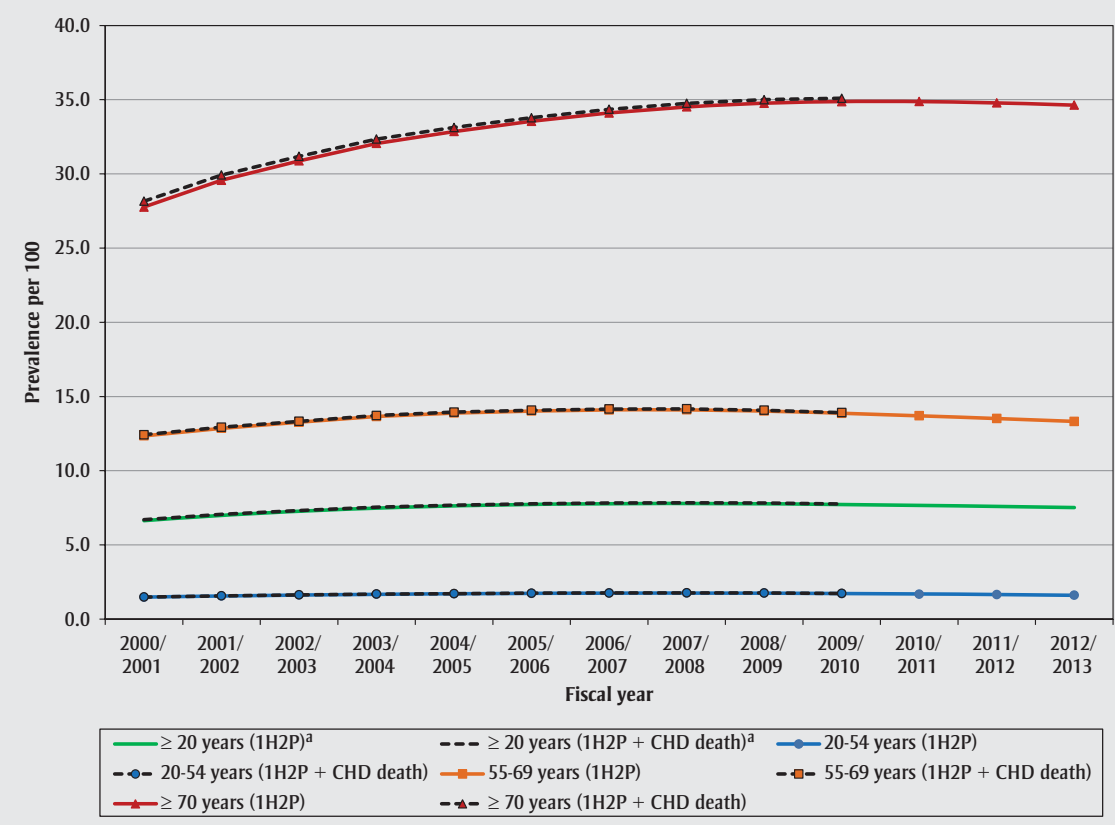

Source: Quebec Integrated Chronic Disease Surveillance System (QICDSS) of the Institut national de santé publique du Québec Abbreviations: $1 \mathrm{H} 2 \mathrm{P}$, one hospital or $\geq 2$ physician claims; CHD, coronary heart disease.

${ }^{a}$ Age standardization using 2001 Quebec Census data as the standard population.

FIGURE 2C

Age-standardized prevalence of diagnosed coronary heart disease, in adults $\geq 20$ years, by sex and age group, Quebec, 2000/2001 to 2012/2013, plus cases identified with coronary heart disease death

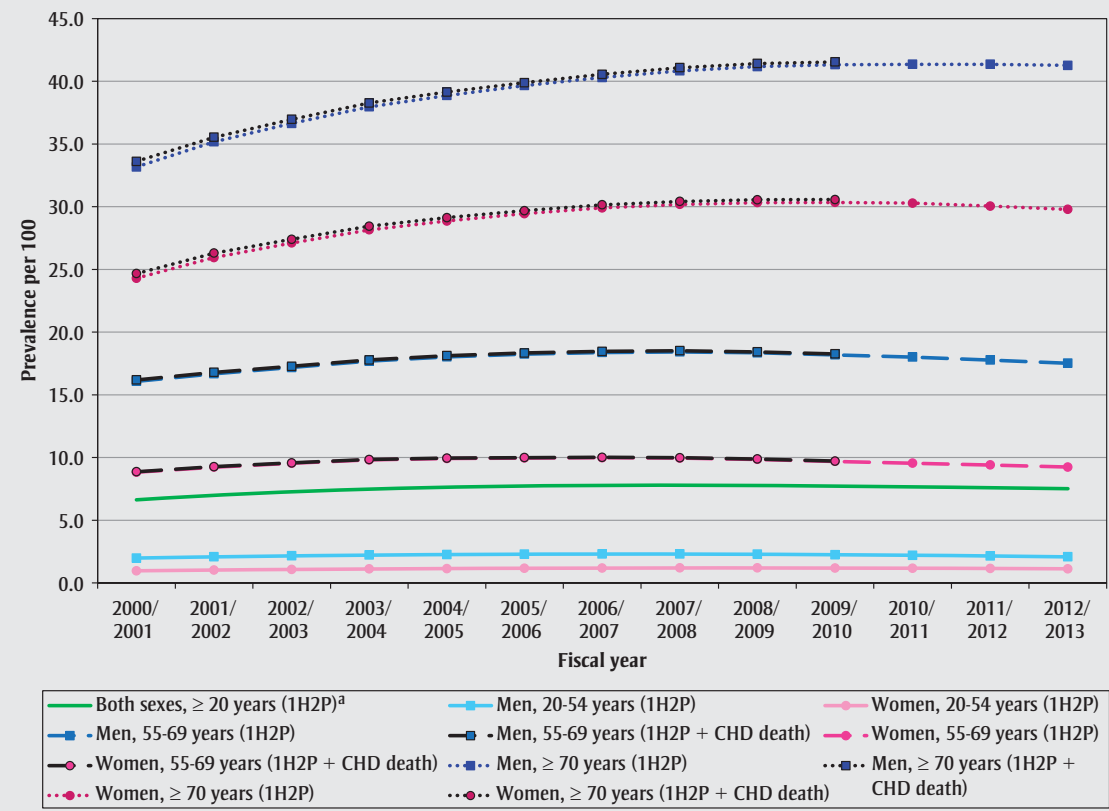

Source: Quebec Integrated Chronic Disease Surveillance System (QICDSS) of the Institut national de santé publique du Québec. Abbreviations: 1H2P, one hospital or $\geq 2$ physician claims; CHD, coronary heart disease.

Note: The addition of the cause of death was not shown for the age group 20-54 years because there is no difference for the two case definitions.

${ }^{\text {a }}$ Age standardization using 2001 Quebec Census data as the standard population. the 2001 Quebec Census population aged 20 years and over as the standard population. Relative changes over time and relative difference between sexes were calculated as previously described ${ }^{7} ; 95 \%$ confidence intervals (CI) were computed using an inverse gamma distribution. Given the populationbased nature of the study, many of the CIs were small and therefore not included in graphs. When the CIs do not overlap, the difference was considered as statistically significant, although this test is considered conservative. Statistical analyses were performed using SAS Enterprise Guide version 5.1 (SAS Institute Inc., Cary, NC, USA).

\section{Results}

\section{Prevalence}

\section{Trends for $1 \mathrm{H} 2 \mathrm{P}$}

In 2012/2013, more than 593000 Quebecers aged 20 years and over were diagnosed with CHD, which represents a crude prevalence of 9.4\% (593 035/6 342 005; 95\% CI: 9.3-9.4). Between 2000/2001 and 2012/2013, the agestandardized prevalence increased by $14 \%$ among men and women although it has decreased slightly since 2009/2010 (Figure 2A). Over the study period, men had a higher prevalence than women; in 2012/2013, the prevalence in women was $40 \%$ lower than in men.

Trends for the 1H2P + CHD death The additional data from the mortality registry-those who died of CHD without having had a previous diagnosis-added only a very small proportion of cases (statistically significant only in 2000/2001) to those already identified (Figure 2A). This proportion decreased during the study period, from the age-standardized rate of $0.07 \%$ (95\% CI: $0.06 \%-0.07 \%$ ) for the total adult population in $2000 / 2001$ to $0.04 \%$ ( $95 \% \mathrm{CI}$ : $0.04 \%-0.04 \%$ ) in 2009/2011 (see Table 1A).

Trends for each case definition based on age Using the $1 \mathrm{H} 2 \mathrm{P}$ definition, the prevalence of $\mathrm{CHD}$ in people aged 70 years and over increased the most between 2000/2001 and $2009 / 2010$ and slightly decreased afterwards, while this prevalence decreased in the other age groups between 2008/2009 and 2012/2013 (Figure 2B). Including CHD deaths (1H2P + CHD death) added only a 
FIGURE 3A

Age-standardized ${ }^{\mathrm{a}}$ incidence of diagnosed coronary heart disease, by sex and for all adults $\geq 20$ years, Quebec, 2000/2001 to 2012/2013, plus cases identified with coronary heart disease death

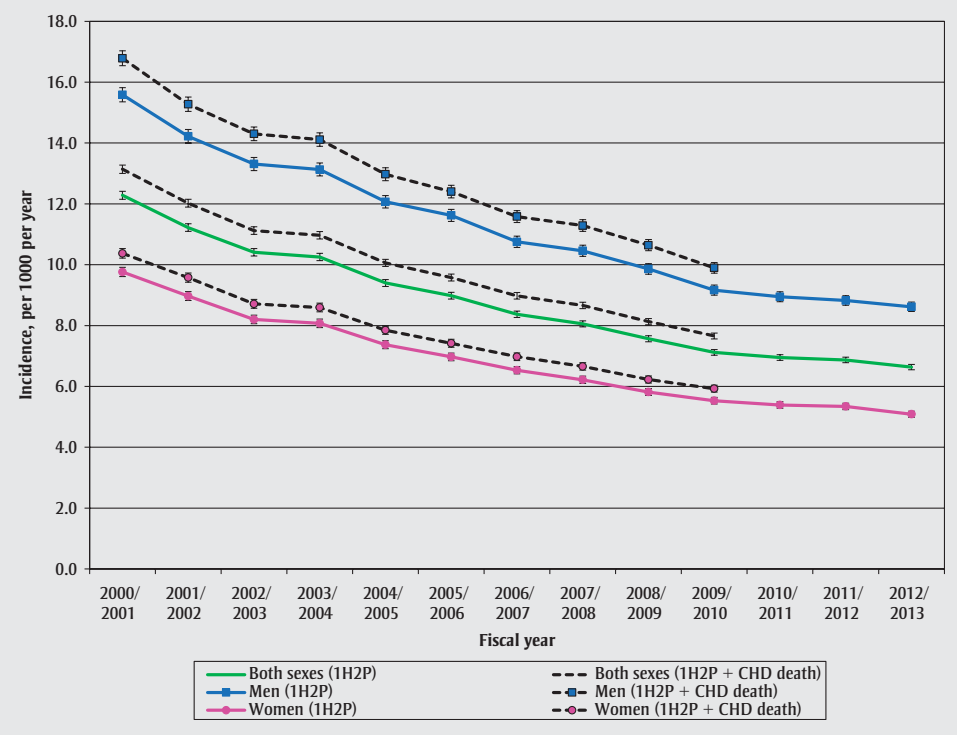

Source: Quebec Integrated Chronic Disease Surveillance System (QICDSS) of the Institut national de santé publique du Québec. Abbreviations: $1 \mathrm{H} 2 \mathrm{P}$, one hospital or $\geq 2$ physician claims; CHD, coronary heart disease.

Note: $95 \%$ confidence intervals are represented by the vertical bars.

${ }^{\text {a }}$ Age standardization using 2001 Quebec Census data as the standard population.

FIGURE 3B

Age-standardized incidence of diagnosed coronary heart disease, by age group, in adults $\geq 20$ years, Quebec, 2000/2001 to 2012/2013, plus cases identified with coronary heart disease death

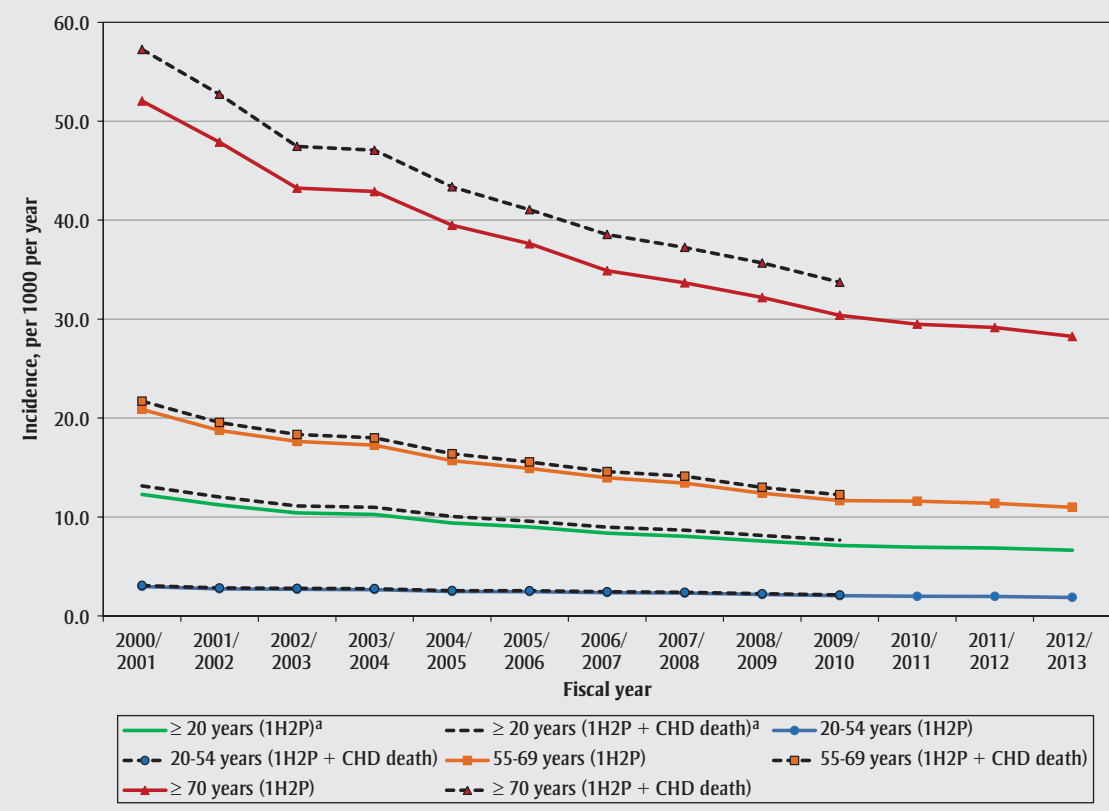

Source: Quebec Integrated Chronic Disease Surveillance System (QICDSS) of the Institut national de santé publique du Québec. Abbreviations: $1 \mathrm{H} 2 \mathrm{P}$, one hospital or $\geq 2$ physician claims; CHD, coronary heart disease.

${ }^{a}$ Age standardization using 2001 Quebec Census data as the standard population. few cases, which was barely significant in the first two years, 2000/2001 and 2001/ 2002, for people aged 70 years and more. Regardless of age, the prevalence in men was continually higher over this period (Figure 2C).

\section{Incidence}

\section{Trends for $1 \mathrm{H} 2 \mathrm{P}$}

In 2012/2013, nearly 40000 people were diagnosed with CHD for the first time, making the crude incidence rate 6.9 per 1000 (39 850/5 788 825; $95 \%$ CI: 6.8-7.0). Between 2000/2001 and 2012/2013, the agestandardized incidence of CHD decreased by $46 \%$ for both sexes combined (Figure $3 \mathrm{~A}$ ). Over this period, women had a lower incidence of CHD than did men, by as much as $41 \%$ in $2012 / 2013$.

\section{Trends for 1H2P + CHD death}

Taking into account CHD as the cause of death significantly increased the absolute incidence of CHD by an average of 0.7 between 2000/2001 and 2009/2010 for both sexes combined (Figure 3A). The proportion identified through CHD death only decreased over time and was higher in men (Table 1B).

Trends for each case definition based on age Based on the 1H2P definition, the incidence of CHD decreased over time and particularly for those aged 70 years and over (Figure 3B). Also taking into account CHD deaths (the $1 \mathrm{H} 2 \mathrm{P}+\mathrm{CHD}$ death definition) added a significant proportion to the incidence for the oldest age group ( $\geq 70$ years) only: the incidence increased from 30.4 per $1000(95 \%$ CI: $29.8-31.0 ; \mathrm{n}=17400$ cases) in 2009/ 2010 to 33.7 per 1000 (95\% CI: 33.1-34.4; $\mathrm{n}=19310$ cases). As with prevalence, incidence was always higher in men than in women in all the age group (Figure 3C).

\section{All-cause mortality}

\section{Trends for $1 \mathrm{H} 2 \mathrm{P}$}

Over 30000 people diagnosed with CHD died in $2012 / 2013$. These people had been hospitalized or had consulted a physician for CHD, and a date of death was recorded in their insurance registry (all causes of death). This represented a crude proportion of 5.2\% (30 550/593 035; 95\% CI 5.1-5.2). Figure $4 \mathrm{~A}$ shows that the adjusted mortality 


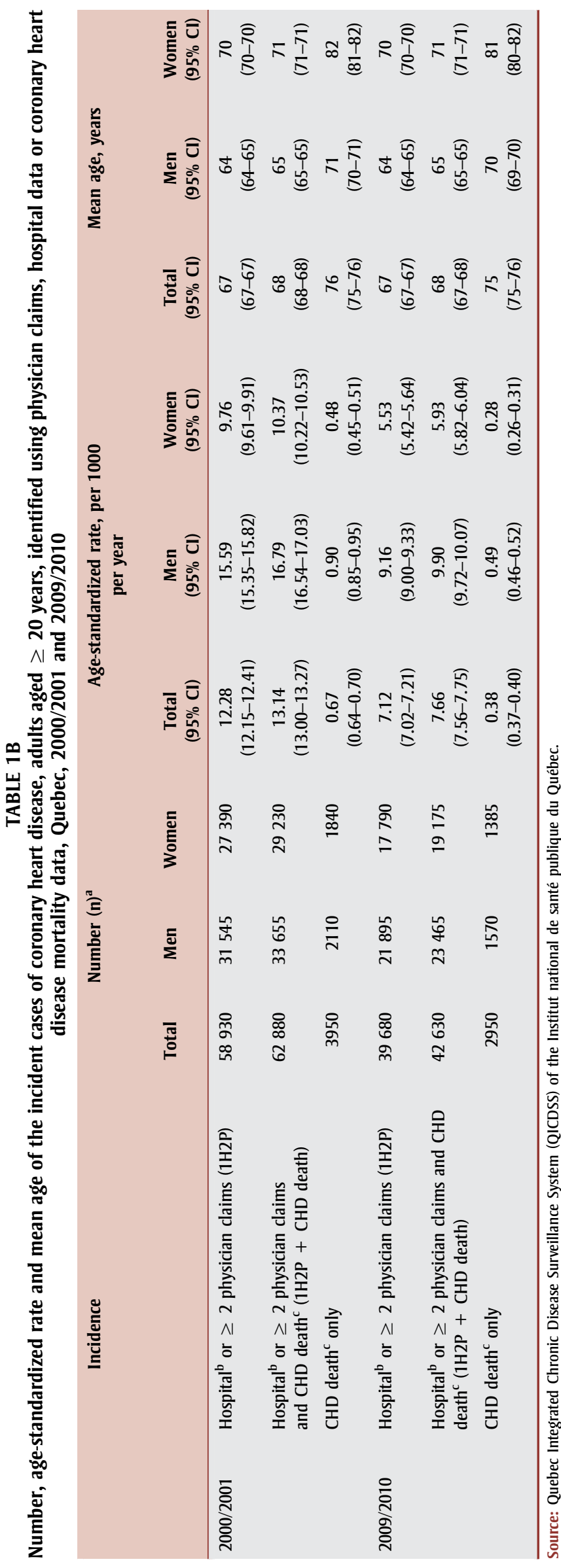

rate decreased for both sexes combined by $26 \%$ between 2000/2001 and 2012/2013.

Trends for 1H2P + CHD death

In 2009/2010, taking into account CHD deaths among people not previously diagnosed with CHD in addition to all causes of death for prevalent cases increased the age-standardized mortality rate, although this increase was insignificant (Table $1 \mathrm{C}$ and Figure 4A). As with prevalence and incidence, this proportion decreased over time.

Sex and age group trends for each case definition

Using either case definition, men and women had similar mortality rates, particularly since 2008/2009 (Figure 4B). Between 2000/2001 and 2009/2010, the addition of CHD death increased average mortality rates nonsignificantly by 0.3 and 0.6 in absolute values, for women and men respectively. Overall mortality rates of the three age groups declined (see Figure 4C). Adding CHD as the cause of death significantly increased mortality rates for all age groups, although it did not have an effect on trends. Mortality rates were very similar in both sexes and all age groups (data not shown).

\section{Mean age for prevalence, incidence and mortality based on sex}

For prevalence and incidence, both sexes combined, the mean age of patients identified through CHD death only was consistently significantly higher than that of patients identified through physician claims or hospital data over the study period (see Tables $1 \mathrm{~A}$ and $1 \mathrm{~B}$, respectively). This age difference was driven by the one observed in women (11 years older in 2009/2010 for incident cases in women, compared to 6 years older for men). However, in the case of mortality rates, the people identified with CHD death only were younger. This difference was most pronounced in men (8 years younger in $2009 / 2010$, compared to 2 years younger for women; see Table $1 \mathrm{C}$ ).

\section{Discussion}

Based on either case definition, 1H2P (hospital or $\geq 2$ physician claims) or $1 \mathrm{H} 2 \mathrm{P}+\mathrm{CHD}$ death (hospital or $\geq 2$ physician claims and CHD death), the prevalence of CHD tended to 
FIGURE 3C

Age-standardized incidence of diagnosed coronary heart disease in adults $\geq 20$ years, by sex and age group, Quebec, $2000 / 2001$ to $2012 / 2013$, plus cases identified with coronary heart disease death

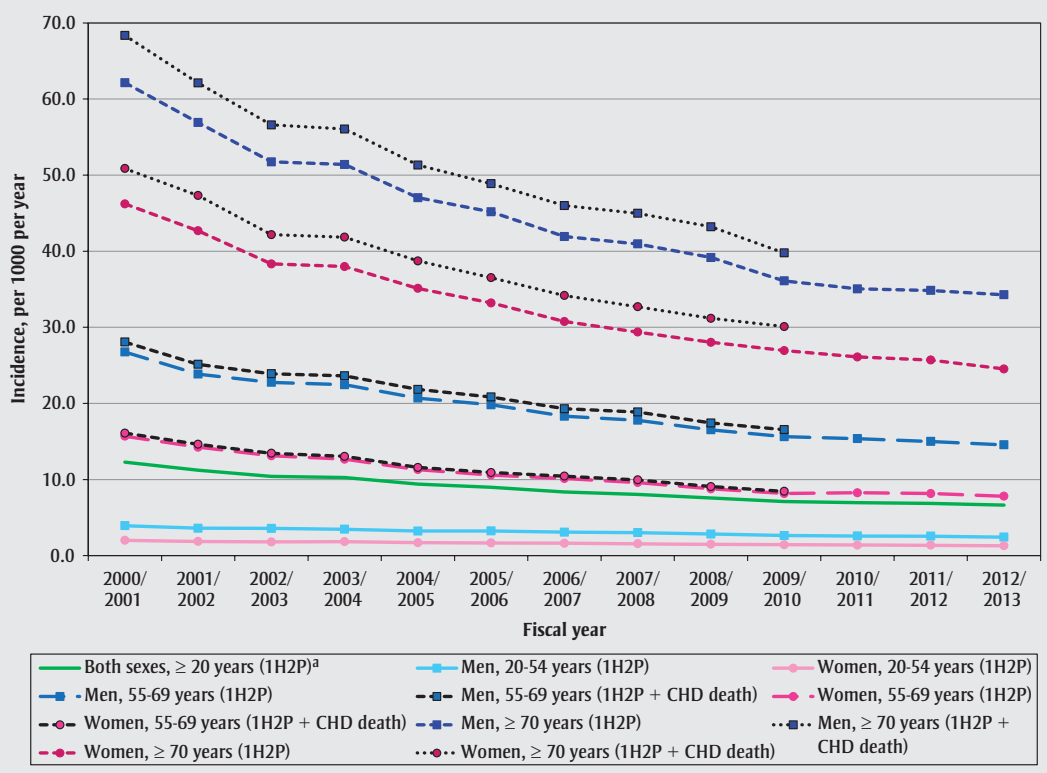

Source: Quebec Integrated Chronic Disease Surveillance System (QICDSS) of the Institut national de santé publique du Québec Abbreviations: $1 \mathrm{H} 2 \mathrm{P}$, one hospital or $\geq 2$ physician claims; $\mathrm{CHD}$, coronary heart disease.

Note: The addition of the cause of death was not shown for the age group 20-54 years because there is no difference for the two case definitions.

${ }^{\text {a }}$ Age standardization using 2001 Quebec Census data as the standard population.

\section{FIGURE 4A}

Age-standardized ${ }^{\mathrm{a}}$ mortality rate for adults aged $\geq 20$ years with diagnosed coronary heart disease, Quebec, 2000/2001 to 2012/2013, plus cases identified with coronary heart disease death

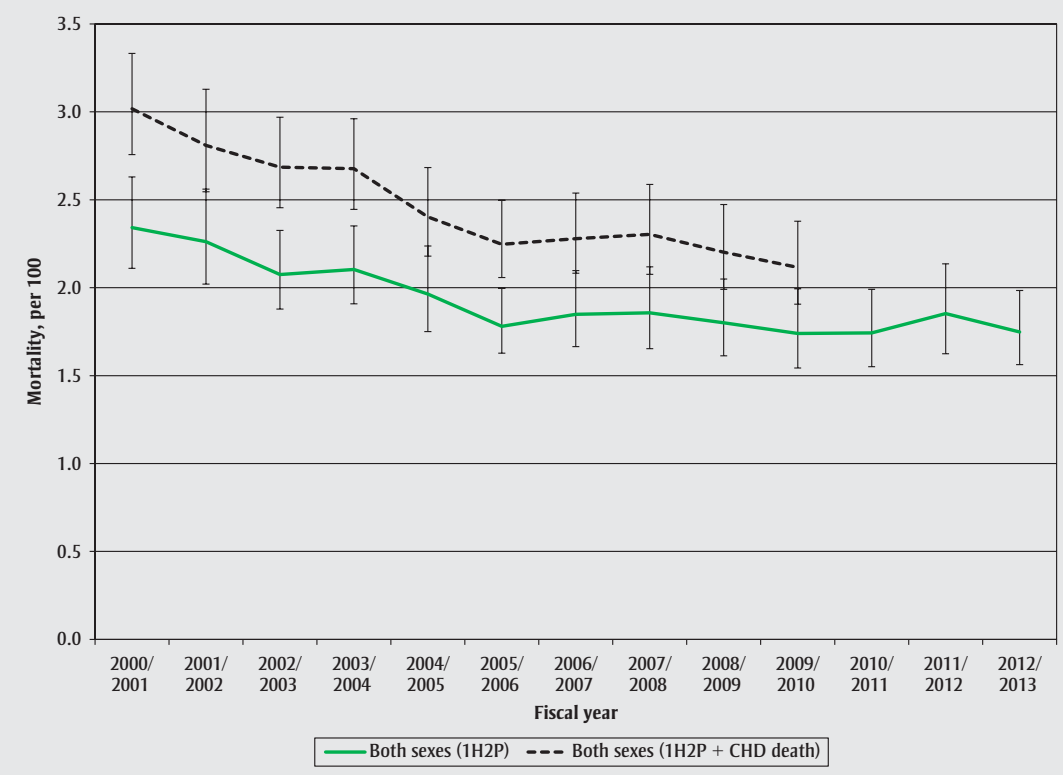

Source: Quebec Integrated Chronic Disease Surveillance System (QICDSS) of the Institut national de santé publique du Québec Abbreviations: $1 \mathrm{H} 2 \mathrm{P}$, one hospital or $\geq 2$ physician claims; CHD, coronary heart disease.

Note: $95 \%$ confidence intervals are represented by the vertical bars.

${ }^{a}$ Age standardization using 2001 Quebec Census data as the standard population. decrease in recent years, while incidence and mortality declined over the study period. The proportions of $\mathrm{CHD}$ mortality that occurred without any previous diagnosis or treatment for CHD, our proxy for SCD, decreased over the study period and were statistically significant for incident cases only, although the differences were small. The incident rate of this proxy for SCD was around 0.38 per 1000 in 2009/2010. Our results thus confirm that a very small proportion of $\mathrm{CHD}$ patients in the province of Quebec died without having been diagnosed with CHD by a physician. Men had a significantly higher prevalence and incidence of $\mathrm{CHD}$ while their mortality rates were about the same as women's.

Our results are similar to those of Moran et al., ${ }^{21}$ who demonstrated that age-standardized incidence of myocardial infarction and prevalence of angina decreased globally between 1990 and 2010 in 21 world regions. Similarly, in most world regions age-standardized CHD mortality rates have declined since 1980, particularly in high-income regions, which is testament to effective prevention and treatment strategies. ${ }^{2}$ The most recent data, from the National Health and Nutrition Examination Survey (NHANES) 2007 to 2010, showed that the prevalence of $\mathrm{CHD}$ in the United States was $6.4 \%$ among adults 20 years and over. ${ }^{22}$ This prevalence is lower than what we found, but this probably reflects underestimation due to the selfreported status of information on the disease.

Shah et al. ${ }^{14}$ studied the temporal trends of risk factor profiles in patients without known cardiovascular disease presenting with a first episode of myocardial infarction, and found modest improvements between 2002 and 2008. The majority of the other studies that focussed on SCD presented a problem by selecting common definitions and criteria, which did not help evaluate incidence. ${ }^{23}$ For example, some studies included time constraints in their case definitions, others included a geographical location of the event or "survivors of cardiac arrest;" most important were the differences in the criteria, from using CHD death only to including cardiovascular etiology. A recent study that used multiple sources of information, such as a death certificate, county, state and national population data, and a prospective population-based surveillance study of SCD, revealed that the 


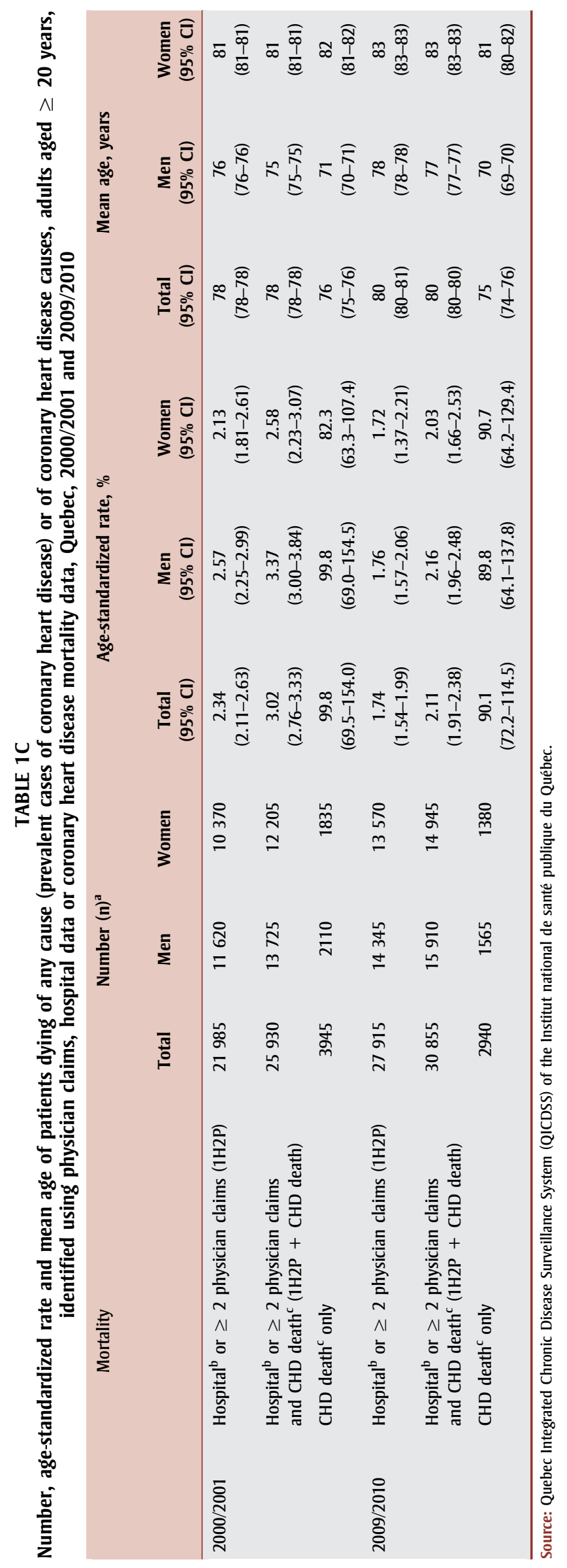

age-adjusted incidence of SCD in the United States was 60 per 100000 population in 2009 , which is similar to what we found in Quebec. $^{24}$ In parallel with the decline in CHD mortality, trends in the incidence of SCD also declined. ${ }^{25}$

\section{Limitations}

Using retrospective health administrative data to estimate the burden of diseases presents many previously described limitations: ${ }^{7,8,17,18,20}$ cases in nursing homes or other institutions may be underestimated; physicians paid through alternative methods; or identified cases are limited to people in contact with the health care system. However, some of these limitations have less impact in the case of CHD, which is often treated in hospitals and it is usually symptomatic. The case definition of CHD maximizes specificity $(97.5 \%)$ and negative predictive value $(97.7 \%)$, while sensitivity $(77.0 \%)$ and positive predictive value (PPV) $(75.3 \%)$ are not as high. ${ }^{19}$ It can be difficult to accurately determine $\mathrm{CHD}$ as the cause of death, particularly if there is no prior history of $\mathrm{CHD}$ or if no autopsy is performed. Nonetheless, the accuracy of death certificates has been validated in the Framingham Heart Study ${ }^{26}$ and Atherosclerosis Risk in Communities $^{27}$ (ARIC) cohort studies, and PPV was $67 \%$ in both studies. These studies found that death due to $\mathrm{CHD}$, based on death certificates, was overestimated by $24 \%$ and $20 \%$, respectively. However, even with this overestimation, our prevalent cases of $\mathrm{CHD}$ are not affected by the addition of CHD death. Our proxy of SCD, silent CHD, can be overestimated, because patients could have had diabetes, hypertension or any heart disease that could be related and could explain their CHD death.

\section{Strengths}

Because Quebec has universal health care, access to treatment for patients with symptoms suggestive of $\mathrm{CHD}$ should be equal. ${ }^{28}$ We used a validated definition of CHD with both diagnosis and treatment codes, which increases the sensitivity and specificity, and relies more on hospitalization data, which have been proven to be useful and reliable. ${ }^{29}$ The QICDSS has all the health information about several chronic diseases for almost the 
FIGURE 4B

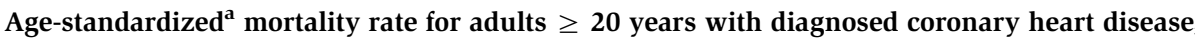
by sex, Quebec, 2000/2001 to 2012/2013, plus cases identified with coronary heart disease death

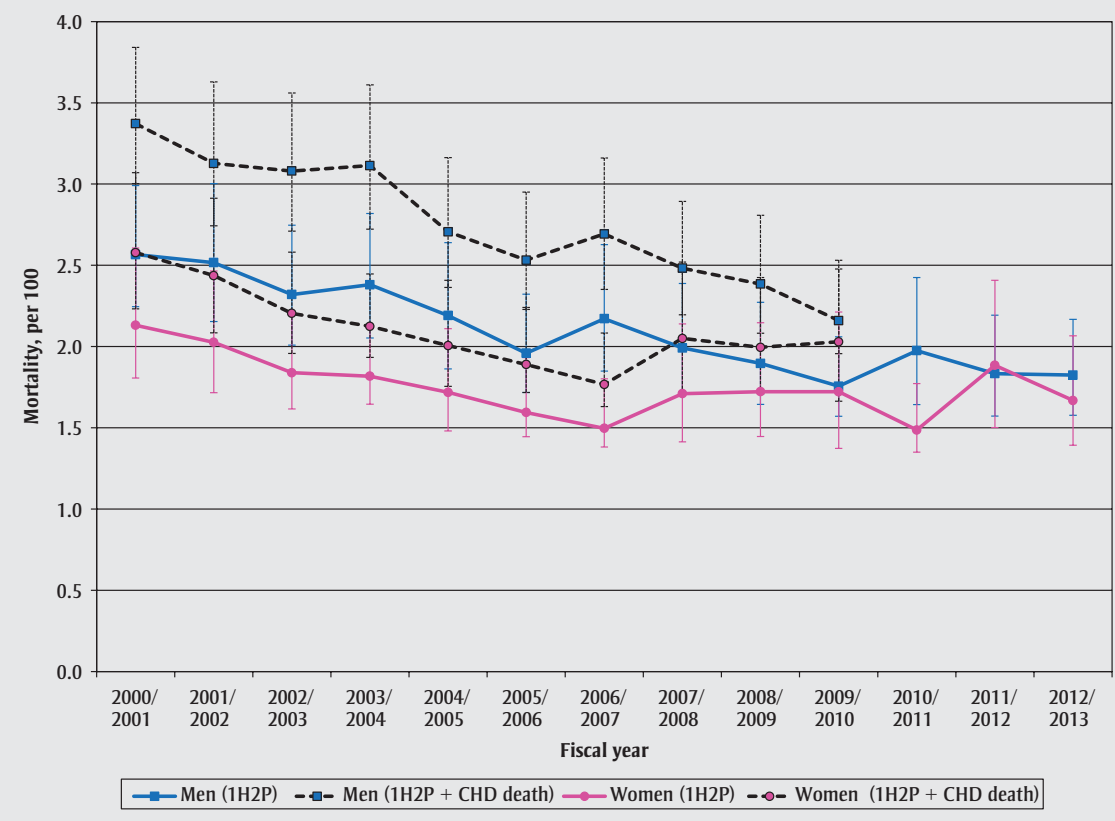

Source: Quebec Integrated Chronic Disease Surveillance System (QICDSS) of the Institut national de santé publique du Québec Abbreviations: 1H2P, one hospital or $\geq 2$ physician claims; CHD, coronary heart disease. Note: $95 \%$ confidence intervals are represented by the vertical bars.

${ }^{a}$ Age standardization using 2001 Quebec Census data as the standard population.

FIGURE 4C

Age-standardized mortality rate for adults $\geq 20$ years with diagnosed coronary heart disease, by age group, Quebec, 2000/2001 to 2012/2013, plus cases identified with coronary heart disease death

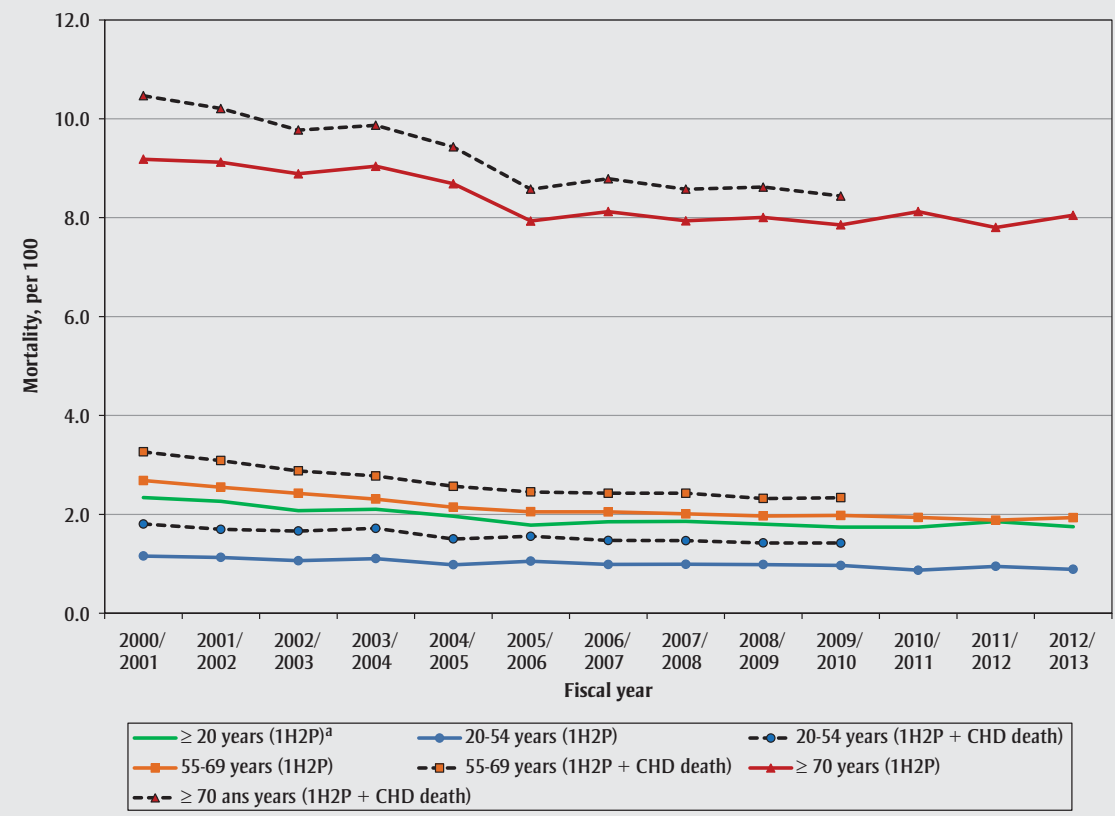

Source: Quebec Integrated Chronic Disease Surveillance System (QICDSS) of the Institut national de santé publique du Québec. Abbreviations: $1 \mathrm{H} 2 \mathrm{P}$, one hospital or $\geq 2$ physician claims; CHD, coronary heart disease.

a Age standardization using 2001 Quebec Census data as the standard population. entire Quebec population (95\% in 2011/ $2012^{17}$ ) making our data very representative. This surveillance system has already gathered more than 15 years of data, and as it is an on-going process, future trends can be easily determined and health services adjusted accordingly.

Quebec is one of the few provinces in Canada that can link vital statistics with other health administrative data. ${ }^{18}$ As a result, our study is strengthened by including the numbers of cases who died of CHD before receiving a diagnosis.

Finally, our proxy of SCD is enhanced by the fact that the majority ( $62 \%$ ) of young people with SCD experienced angina. ${ }^{30}$ This means that CHD diagnosis, which includes angina, was likely present before death. This proxy is also the most instinctive definition, because it answers one of the first questions that arise when a person dies suddenly: "Was this person at risk or had any history of CHD?"

\section{Conclusion}

The decreasing trends in CHD are encouraging. The proportion identified only through vital statistics is also decreasing and very small. This proxy of SCD represents an insignificant proportion of $\mathrm{CHD}$, as illustrated with the prevalence. We can suppose that secondary prevention has been beneficial. Primary prevention of $\mathrm{CHD}$ should be reinforced, as some cases were only identified when death from CHD occurred, particularly among the elderly. However, because we are confirming that a very small proportion of Quebecers were dying suddenly from $\mathrm{CHD}$, a further methodological implication is that claims and hospital data are sufficient to perform CHD surveillance. Because all Canadian provinces and territories have a similar universal health care system, we can extrapolate that $\mathrm{CHD}$ surveillance in Canada can be done with these two databases alone. ${ }^{18}$ Nonetheless, future studies about silent CHD should include the place of death as well as the history of other diagnoses in order to specify who is more at risk. Public health advice should also emphasize consultation for cardiovascular diseases and its risk factors, that is, primary prevention, as the best way to further improve trends in $\mathrm{CHD}$ and SCD. 


\section{Acknowledgements}

The authors wish to thank the Public Health Agency of Canada and the Ministère de la Santé et des Services sociaux du Québec for their financial support.

\section{References}

1. Mendis S, Puska P, Norrving B. Global Atlas on Cardiovascular Disease Prevention and Control. 1-155Geneva (CH): World Health Organization; 2011

2. Moran AE, Forouzanfar MH, Roth G, et al. Temporal trends in ischemic heart disease mortality in 21 world regions, 1980 to 2010 : the Global Burden of Disease 2010 Study. Circulation. 2014;129(14):1483-92.

3. Tunstall-Pedoe $H$, Vanuzzo D, Hobbs $M$, et al. Estimation of contribution of changes in coronary care to improving survival, event rates, and coronary heart disease mortality across the WHO MONICA Project populations. Lancet. 2000;355(9205):688-700.

4. Kuulasmaa K, Tunstall-Pedoe H, Dobson A, et al. Estimation of contribution of changes in classic risk factors to trends in coronaryevent rates across the WHO MONICA Project populations. Lancet. 2000;355(9205):675-87.

5. Ford ES, Ajani UA, Croft JB, et al. Explaining the decrease in U.S. deaths from coronary disease, 1980-2000. N Engl J Med. 2007;356(23):2388-98.

6. Wijeysundera HC, Machado M, Farahati F, et al. Association of temporal trends in risk factors and treatment uptake with coronary heart disease mortality, 1994-2005. JAMA. 2010;303(18):1841-7.

7. Blais C, Rochette L, Hamel D, Poirier P. Prevalence, incidence, awareness and control of hypertension in the province of Quebec: Perspective from administrative and survey data. Can J Public Health. 2014;105(1):e79-85.

8. Robitaille C, Dai S, Waters C, et al. Diagnosed hypertension in Canada: incidence, prevalence and associated mortality. CMAJ. 2012;184(1):E49-E56.

9. Pelletier C, Dai S, Roberts KC, Bienek A, Onysko J, Pelletier L. Repssort summary. Diabetes in Canada: facts and figures from a public health perspective. Chronic Dis Inj Can. 2012;33(1):53-4.
10. Padwal RS. Obesity, diabetes, and the metabolic syndrome: the global scourge. Can J Cardiol. 2014;30(5):467-72.

11. Goraya TY, Jacobsen SJ, Kottke TE, Frye RL, Weston SA, Roger VL. Coronary heart disease death and sudden cardiac death: a 20-year population-based study. Am J Epidemiol. 2003;157(9):763-70.

12. Salomaa V, Ketonen M, Koukkunen $\mathrm{H}$, et al. Decline in out-of-hospital coronary heart disease deaths has contributed the main part to the overall decline in coronary heart disease mortality rates among persons 35 to 64 years of age in Finland: the FINAMI study. Circulation. 2003;108(6):691-6.

13. Arciero TJ, Jacobsen SJ, Reeder GS, et al. Temporal trends in the incidence of coronary disease. Am J Med. 2004;117(4):228-33.

14. Shah B, Bangalore S, Gianos E, et al. Temporal trends in clinical characteristics of patients without known cardiovascular disease with a first episode of myocardial infarction. Am Heart J. 2014;167(4):480-88.

15. Henkel DM, Witt BJ, Gersh BJ, et al. Ventricular arrhythmias after acute myocardial infarction: a 20-year community study. Am Heart J. 2006;151(4):806-12.

16. Chugh SS, Reinier K, Teodorescu C, et al. Epidemiology of sudden cardiac death: clinical and research implications. Prog Cardiovasc Dis. 2008;51(3):213-28.

17. Blais C, Jean S, Sirois C, et al. Quebec Integrated Chronic Disease Surveillance System (QICDSS), an innovative approach. Chronic Dis Inj Can. 2014;34(4):226-35.

18. Robitaille C, Bancej C, Dai S, et al. Surveillance of ischemic heart disease should include physician billing claims: populationbased evidence from administrative health data across seven Canadian provinces. BMC Cardiovasc Disord. 2013;13(1):88.

19. Tu K, Mitiku T, Lee DS, Guo H, Tu JV. Validation of physician billing and hospitalization data to identify patients with ischemic heart disease using data from the Electronic Medical Record Administrative Data Linked Database (EMRALD). Can J Cardiol. 2010;26(7):e225-8.

20. Blais C, Dai S, Waters C, et al. Assessing the burden of hospitalized and community-care heart failure in Canada. Can J Cardiol. 2014;30(3):352-8.
21. Moran AE, Forouzanfar MH, Roth G, et al. The global burden of ischemic heart disease in 1990 and 2010: the Global Burden of Disease 2010 study. Circulation. 2014;129(14): 1493-501.

22. Go AS, Mozaffarian D, Roger VL, et al. Heart disease and stroke statistics-2014 update: a report from the American Heart Association. Circulation. 2014;129(3):e28-292.

23. Kong MH, Fonarow GC, Peterson ED, et al. Systematic review of the incidence of sudden cardiac death in the United States. J Am Coll Cardiol. 2011;57(7):794-801.

24. Stecker EC, Reinier K, Marijon E, et al. Public health burden of sudden cardiac death in the United States. Circ Arrhythm Electrophysiol. 2014;7 (2):212-7.

25. Ni H, Coady S, Rosamond W, et al. Trends from 1987 to 2004 in sudden death due to coronary heart disease: the Atherosclerosis Risk in Communities (ARIC) study. Am Heart J. 2009;157(1):46-52.

26. Lloyd-Jones DM, Martin DO, Larson MG, Levy D. Accuracy of death certificates for coding coronary heart disease as the cause of death. Ann Intern Med. 1998;129(12): 1020-6.

27. Coady SA, Sorlie PD, Cooper LS, Folsom AR, Rosamond WD, Conwill DE. Validation of death certificate diagnosis for coronary heart disease: the Atherosclerosis Risk in Communities (ARIC) Study. J Clin Epidemiol. 2001;54(1):40-50.

28. Blais C, Hamel D, Rinfret S. Impact of socioeconomic deprivation and area of residence on access to coronary revascularization and mortality after a first acute myocardial infarction in Quebec. Can J Cardiol. 2012;28(2):169-77.

29. Lambert L, Blais C, Hamel D, et al. Evaluation of care and surveillance of cardiovascular disease: can we trust medicoadministrative hospital data? Can J Cardiol. 2012;28(2):162-8.

30. Jabbari R, Risgaard B, Holst AG, et al. Cardiac symptoms before sudden cardiac death caused by coronary artery disease: a nationwide study among young Danish people. Heart. 2013;99(13):938-43. 